\title{
Genomic and expression array profiling of chromosome 20q amplicon in human colon cancer cells
}

\author{
Jennifer I Carter, Li Jin, ${ }^{1}$ Subrata Sen \\ University of Texas - M. D. Anderson Cancer Center, ${ }^{1}$ PD, University of Cincinnati
}

BACKGROUND: Gain of the q arm of chromosome 20 in human colorectal cancer has been associated with poorer survival time and has been reported to increase in frequency from adenomas to metastasis. The increasing frequency of chromosome $20 \mathrm{q}$ amplification during colorectal cancer progression and the presence of this amplification in carcinomas of other tissue origin has lead us to hypothesize that 20q11-13 harbors one or more genes which, when over expressed promote tumor invasion and metastasis. AIMS: Generate genomic and expression profiles of the $20 \mathrm{q}$ amplicon in human cancer cell lines in order to identify genes with increased copy number and expression.

MATERIALS AND METHODS: Utilizing genomic sequencing clones and amplification mapping data from our lab and other previous studies, BAC/ PAC tiling paths spanning the $20 \mathrm{q}$ amplicon and genomic microarrays were generated. Array-CGH on the custom array with human cancer cell line DNAs was performed to generate genomic profiles of the amplicon. Expression array analysis with RNA from these cell lines using commercial oligo microarrays generated expression profiles of the amplicon. The data were then combined in order to identify genes with increased copy number and expression.

RESULTS: Over expressed genes in regions of increased copy number were identified and a list of potential novel genetic tumor markers was assembled based on biological functions of these genes

CONCLUSIONS: Performing high-resolution genomic microarray profiling in conjunction with expression analysis is an effective approach to identify potential tumor markers.

Key words: Cancer, Array-CGH, Amplification, Genomic Profiling

Cancer, a disease of impaired genomic stability, remains one of the greatest threats to human health and a challenge to medical science in the twenty-first century. Genomic instability has been implicated as providing cancer cells with the ability to undergo clonal evolution facilitating emergence of cells with uncontrolled capacity for proliferation, survival, angiogenesis, inva- sion and metastasis..$^{[1,2]}$ Characterization of genomic rearrangements is, therefore, a major area of investigation being pursued by the cancer research community. Amplification of genomic DNA is one such form of rearrangement that leads to an increase in the copy number of specific genes frequently detected in a variety of human cancer cell types. Our laboratory has been interested in characterizing amplified genomic regions in cancer cells based on the hypothesis that these segments harbor critical genes associated with initiation and/ or progression of cancer. Gain of chromosome $20 q$ in human colorectal cancer has been associated with poorer survival time and has been reported to increase in frequency from around $50 \%$ in adenomas, to $60 \%$ in early carcinomas and to $83 \%$ in late carcinomas and liver metastasis. ${ }^{[3,4,5]}$ The increasing frequency of chromosome $20 \mathrm{q}$ amplification during colorectal cancer progression and the presence of this amplification in carcinomas of diverse origins has lead us to hypothesize that the 20q11-13 amplicon harbors one or more amplified genes which, when over expressed promote tumor invasion and metastasis. In view of this hypothesis we were interested to generate high-resolution amplification profiles for human chromosome $20 \mathrm{q}$ in colorectal cancer cell lines by microarray comparative genomic hybridization (array-CGH) and correlate the results with expression microarray analysis to identify novel amplified and over expressed genes whose function suggest an association with tumor invasion/metastasis.

\section{Material and Methods}

Cell Lines and Culturing: The breast carcinoma cell 
lines MCF7 and SKBr3 were obtained from ATCC and cultured as follows. For MCF7, complete media was DMEM with $2 \mathrm{mM}$ L-glutamine, $5 \mathrm{mM}$ penicillin/streptomycin, fetal bovine serum, 10\%. For SKBr3, complete media was McCoy's 5 a medium with $2 \mathrm{mM} \mathrm{L-glutamine,}$ $5 \mathrm{mM}$ penicillin/streptomycin, fetal bovine serum, $10 \%$. The colon carcinoma cell lines HT29 and Caco-2 cells were obtained from ATCC and cultured as follows. For HT-29 complete media was DMEM with $2 \mathrm{mM} \mathrm{L-}$ glutamine, $5 \mathrm{mM}$ penicillin/streptomycin, fetal bovine serum, 10\%. For CaCo-2, complete media was EMEM with $2 \mathrm{mM}$ L-glutamine, $1.5 \mathrm{~g} / \mathrm{L}$ sodium bicarbonate, 0.1 $\mathrm{mM}$ non-essential amino acids, $1.0 \mathrm{mM}$ sodium pyruvate, $5 \mathrm{mM}$ penicillin/streptomycin, fetal bovine serum, $20 \%$. All cell lines were cultured in a humidified incubator at a temperature of $37^{\circ} \mathrm{C}$ in $95 \%$ air and $5 \% \mathrm{CO}_{2}$.

Microarray Construction: The $20 \mathrm{q}$ amplicon has previously been defined based on FISH analysis in our lab and by others, as the region between STS markers D20S1076 and Z94462. ${ }^{[6]}$ Chromosome 20 has been sequenced primarily by the Wellcome Trust Sanger Institute and the contigs used in the sequencing are available on the WEB at the National Center for Biotechnology Information (NCBI) site. ${ }^{[7]}$ Based on the clones in the sequencing contig NT 011362.7, first a $12 \mathrm{Mb}$ BAC/ PAC contig comprising of 137 clones and then an approximately $22.8 \mathrm{Mb}$ BAC/PAC contig comprising of 253 $\mathrm{BAC}$ and PAC clones were generated from the following human genomic libraries: RPCl-11, RPCl-5, RPCl1, RPCI-3, RPCl-4, and CalTech D2. Figure 1. is a graphical representation of both contigs and their boundaries. The array printing was done in collaboration with the laboratory of Dr. Li Jin at the University of Cincinnati. For array printing, DNA from clones were prepared using the Qiagen Midi Kit (Valencia, CA) and amplified by PCR using human specific degenerate primers designed to eliminate vector DNA, reduce amplification of repetitive sequences and incorporate amino-modified nucleotide. The amplified DNA was micro-spotted with an Omnigrid Microarrayer using the touch pin method onto aldehyde-coated slides. The slides were then crosslinked, washed, denatured and the unbound aldehyde groups blocked.

Labeling Genomic DNA and Array-CGH: Genomic DNA was isolated using the Qiagen DNeasy DNA Extraction Kit according to the manufacturer's protocols.
Sample DNA and reference genomic DNA (Promega, Madison, WI) labeling was carried out similar to previous methods. ${ }^{[8]}$ Genomic DNA was digested with the endonuclease $\alpha$ Taq I, quantitated and examined for complete digestion by agarose gel electrophoresis. Random priming reactions of DNA using BioPrime Random Labeling Kit (Invitrogen, Carlsbad, CA) were carried out according to manufacturer's protocol with: $2 \mathrm{nmol}$ dATP, dGTP, dCTP, $0.6 \mathrm{nmol}$ dTTP and $1.4 \mathrm{nmol}$ of Cy3-dUTP or Cy5-dUTP used in the place of the dNTP

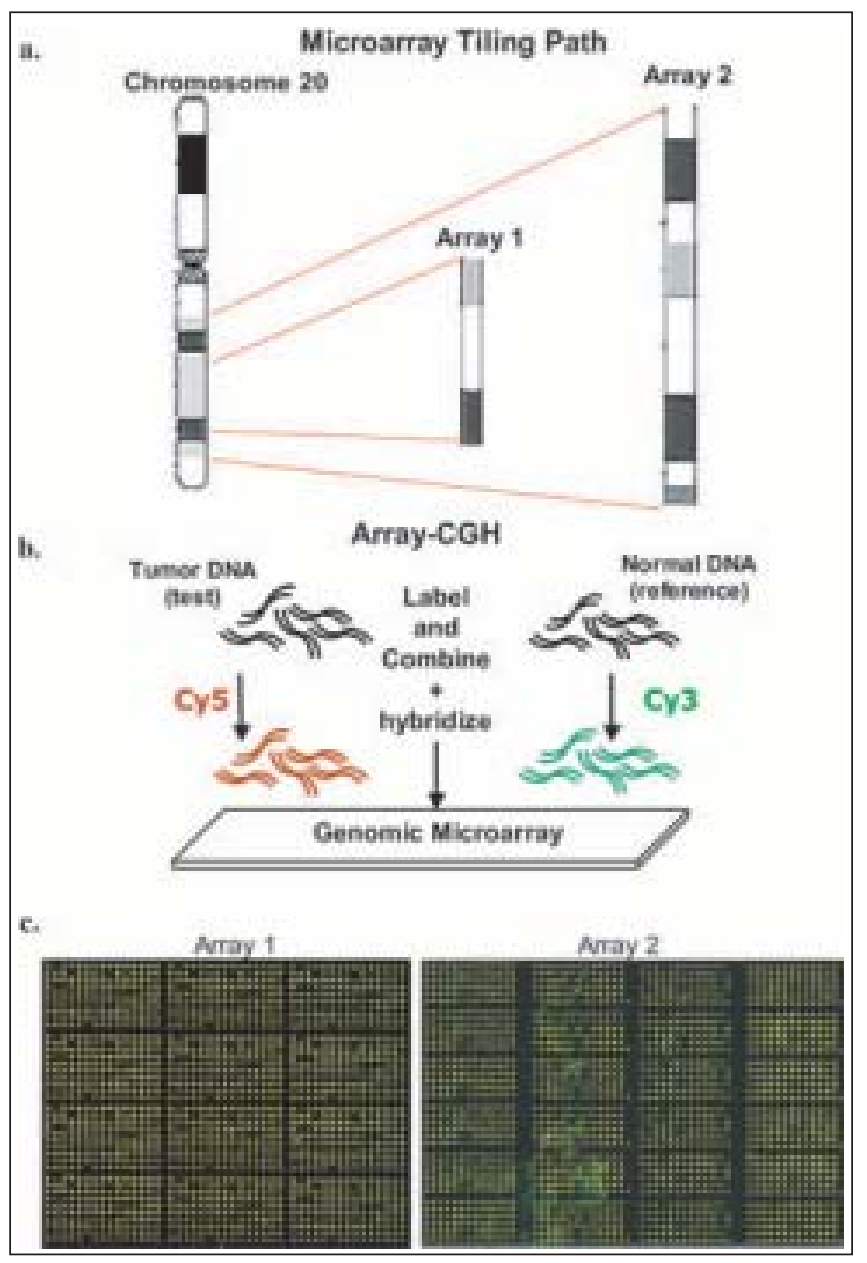

Figure 1a: Representation of the genomic sequencing clone tiling path found on the $\mathrm{NCBI}$ website ${ }^{[7]}$ used to compose the two chromosome 20q BAC $\backslash P A C$

microarrays. Array 1 spans $12.8 \mathrm{MB}$, which reside entirely within the $22 \mathrm{Mb}$ tiling path of Array 2. Figure $1 \mathrm{~b}$ : Visual summary of the array-CGH process showing the differential labeling of test and reference DNA and hybridization. The ratio of Cy5 to Cy3 fluorescence intensity is used to determine the ratio of test to reference DNA. c. Scanned images of array 1 and array 2 control (normal vs normal) hybridizations. Test and reference are the same DNA, the signal for the arrayed elements in each channel (green for Cy3 and red for Cy5) are the same, thus producing the observed yellow signal when channels are combined 
mix provided. Labeled test and standard DNA were combined, COT-1 Human DNA and sheared Salmon sperm DNA were added to block non-specific binding and the mixture was ethanol precipitated. The pellet was resuspended in hybridization buffer and incubated for 1 hour at $42^{\circ} \mathrm{C}$. Hybridization was carried out similarly to previously described methods. ${ }^{[8]}$ The hybridization mixture was deposited on a $22 \mathrm{~mm} X 22 \mathrm{~mm}$ coverslip, placed on the arrayed area of the slide and incubated for 48-72 hours at $42^{\circ} \mathrm{C}$ in a high humidity and light protected environment. Following the incubation the slides were washed for 5 minutes in wash buffer I (2X SSC, 0.5\% SDS), 5 minutes in was buffer II (1X SSC, $0.5 \%$ SDS), 2 minutes in wash buffer III ( $0.2 X$ SSC, $0.3 \%$ SDS) and immediately dried by spinning in a centrifuge. Slides were then visualized by scanning with a GenePix 4000Bi scanner and initially analyzed using Axon GenePix Pro 3.0 software for fluorescence ratios. For each cell line, hybridizations were repeated with inverse labeled test and standard DNA (dye-swap hybridization).

Expression Microarray Analysis: Expression Array analysis: Total cellular RNA was isolated from the cell lines using TRIZOL (Invitrogen, Carlsbad, CA) and further purified using the Qiagen RNeasy RNA Extraction Kit with an on column DNAse digestion. RNA from cell lines and commercially available normal colonic epithelial RNA (BD Biosciences Clonetech, Palo Alto, CA) was labeled using Agilent's Low RNA Input Labeling Kit, which involves reverse transcribing the mRNA to produce cDNA and then transcribing in the presence of Cy3CTP and Cy5-CTP to produce labeled cRNA. The labeled cell line cRNA was paired with the differentially labeled normal cRNA and, using the Agilent In situ Hybridization Kit, hybridized to the Agilent Human $1 \mathrm{~A}$ oligo microarray (v1) or Human 1B oligo microarray according to the manufacturer's protocol. The arrays were scanned on an Agilent DNA microarray scanner and analyzed using Agilent Feature Extraction Software.

Microarray Data Analysis: For array-CGH, background subtracted fluorescence data generated with Genepix software was imported into Excel where replicates were combined and Log base2 ratios calculated. Any array spot with a signal to noise ratio greater than 3 was excluded from the analysis. Normalization was carried out as follows: the average fluorescence ratio across all clones was calculated, each clone's ratio was multiplied by this number and ploidy correction values were added when necessary. Data was graphed in Excel and a moving average per 5 clones was plotted. A log2 ratio of $>0.4$ was considered gain and $>0.8$ considered amplification for this study. For expression arrays, Agilent's Feature Extraction data treatment within the software was as follows: Linear/Lowess normalization was carried out, Log base 10 ratios were calculated and built in error models were used to calculate a $p$-value for each probe. Probes with a signal to noise ratio less than 3 were excluded.

\section{Results}

MCF7 and SKBr3 breast carcinoma cell lines have been extensively characterized in the $20 \mathrm{q}$ region and were used to validate the quality and accuracy of the custom genomic arrays. ${ }^{[9,10]}$ Array-CGH was performed on these cell lines using the initial $12 \mathrm{Mb}$ BAC/PAC genomic array (array 1 ), the resulting genomic profiles are shown in figure 2. The cell line MCF7 showed no copy gain along the array's contig while SKBr3 showed copy gain (log 2 ratio between 0.4 and 0.8 ) from $44-46 \mathrm{Mb}$ and from $52-55 \mathrm{Mb}$ with a region of amplification ( $\log 2$ ratio greater than 0.8 ) around 52 and $55 \mathrm{Mb}$. A second 22.8 Mb BAC/PAC genomic array (array 2) was constructed to provide greater coverage of the $20 \mathrm{q}$ region. The profiles generated from array-CGH analysis agreed well with the profiles previously generated by array 1 [Figure 2]. While MCF7 showed no copy gain over the extended contig, SKBr3's profile from array 2 showed the same copy gains as that of array 1's profile. In the extended amplicon region, SKBr3 showed copy gain extended from $42 \mathrm{Mb}$ to $46 \mathrm{Mb}$ and identified a high copy gain from $37 \mathrm{Mb}$ to $38 \mathrm{Mb}$. Array-CGH was then carried out with DNA from the colorectal carcinoma cell lines HT29 and CaCo-2 DNA on the second array [Figure 3]. The profiles generated reveal differential copy number variation across the $20 \mathrm{q}$ amplicon for both cell lines. Cell line HT29 showed an overall copy gain of $1(\log 2$ ratio $=0.5$ ) with regions of amplification at 37-39 Mb, $42-45 \mathrm{Mb}, 50-53 \mathrm{Mb}$ and $54-56 \mathrm{Mb}$. There is also a very distinct high-level amplification of +3 copies (total copy number $=5$ ) at $54 \mathrm{Mb}$. CaCo-2's genomic profile was very 
complex, showing an average copy gain of 2 (log 2 ratio $=1)$ with specific regions of amplification of $+2.5(\log$ 2 ratio $=1.25)$ at $49 \mathrm{Mb}, 51 \mathrm{Mb}, 54 \mathrm{Mb}$ and $55.5 \mathrm{Mb}$. Amplifications of +3 copies $(\log 2$ ratio $=1.5)$ were seen at
$39 \mathrm{Mb}, 41 \mathrm{Mb}, 44 \mathrm{Mb}, 45 \mathrm{Mb}$. One region of high amplification with a copy number of $+4(\log 2$ ratio $=2)$ was observed at $43 \mathrm{Mb}$. Regions along $20 \mathrm{q}$ that displayed copy gain in both the cell lines were identified as fol-

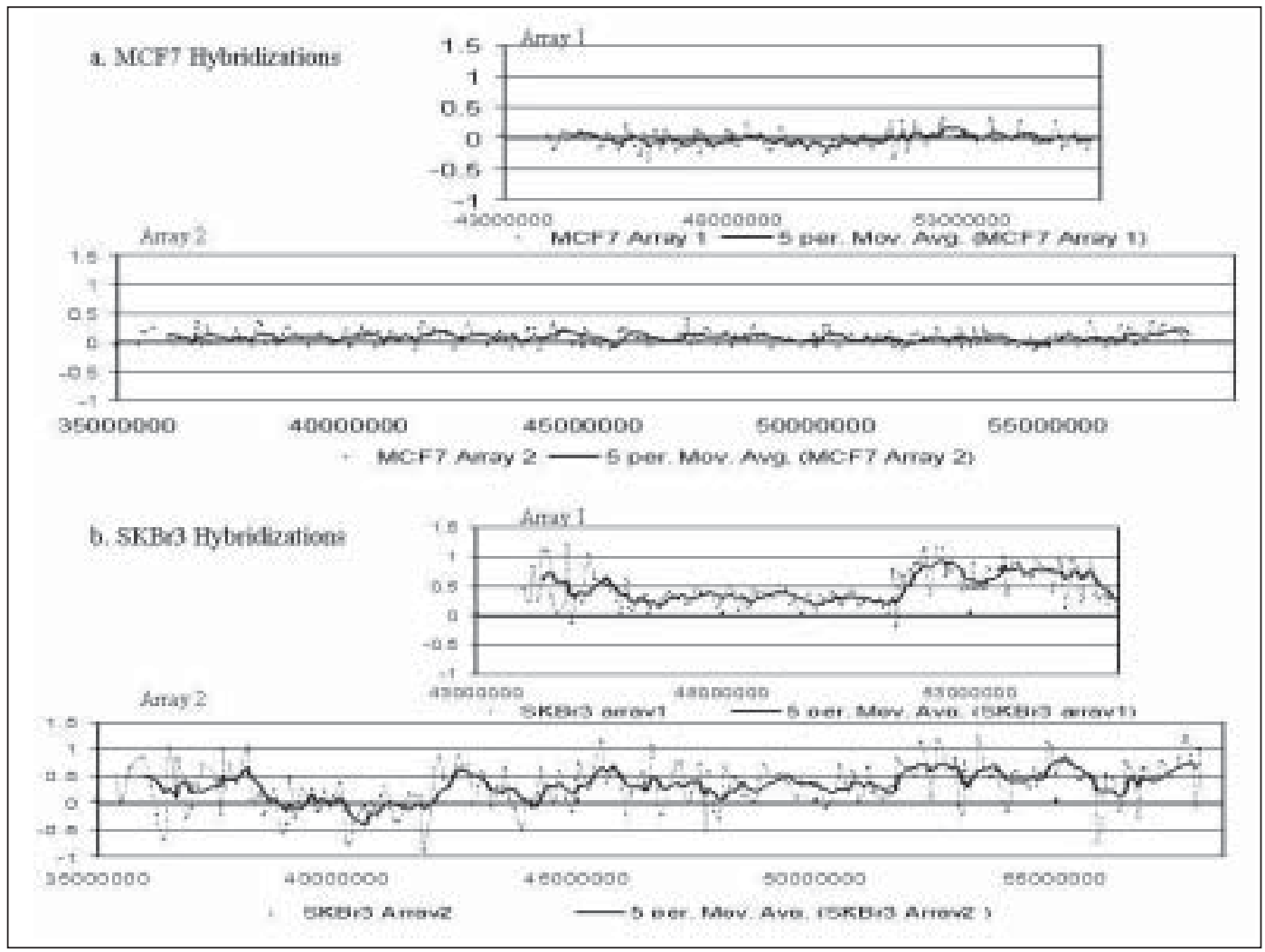

Figure 2: Genomic profile for MCF7 (a.) and SKBr3 (b.) cell lines generated with both array1 and array2. For each graph, the $\mathrm{Y}$-axis unit is Log base2 ratio and the $\mathrm{X}$-axis unit is base pairs along chromosome 20. The Log2 ratio for each clone was plotted at that clones position on chromosome 20 (dotted blue line), and a smoothing curve averaging every 5 data points (black line) was plotted. A Log2 ratio of 0 (red line) represents no change, a ratio of 0.5 represents a gain of one copy, a ratio of 1 represents a gain of 2 copies, etc

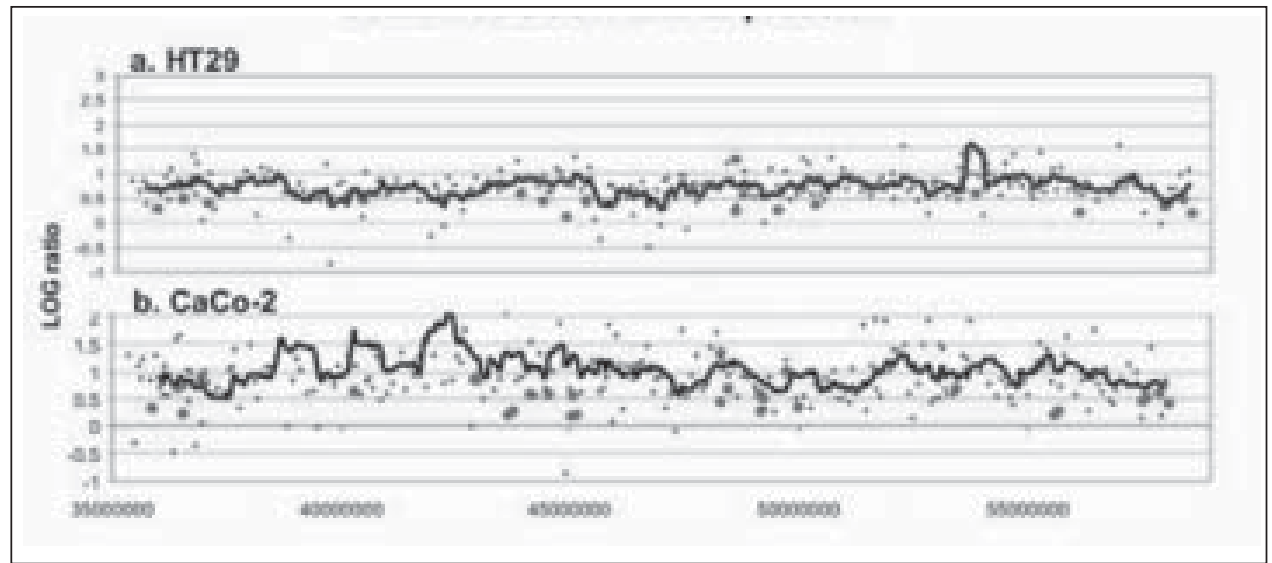

Figure 3: Expression and aCGH profiles for HT29 (a.) and CaCo-2 (b.). For each graph, black dots represent the log 2 ration value of aCGH clones (Array 2) and the black line represents the moving average for every 5 clones, producing a genomic copy number profile. Large red dots represent genes whose log 10 ratio values are significantly increased over normal colonic epithelial cells. The X-axis scale is base pairs along chromosome 20. The y-axis scale is log base 2 for aCGH (in black) and log base 10 (in red) 
lows: 37-39 Mb, 42-45 Mb, 48-50 Mb, and 52-56 Mb.

Subsequent expression analysis using the Agilent Human1A (v1) and Human 1B oligo arrays produced an expression profile for $20 \mathrm{q}$ that showed great variation in the expression of genes from significantly downregulated to significantly up-regulated, although the majority of genes showed no variation in expression between cancer cell line, and normal colonic epithelium. A list of 23 genes for HT29 and 34 genes for CaCo-2 whose probes showed significant $(p<0.01)$ increased expression levels from normal colonic epithelium [Figure3 in red] was generated. Of these genes, 21 were commonly over expressed in both cell lines. Taking into account the genomic copy number profile as well as the known function of the gene product, a list of 10 candidate tumor marker genes was generated [Table1].

\section{Discussion}

While in the past most studies of genomic instability in colorectal cancer have focused on deletions and loss of tumor suppressor genes such as APC, DCC and p53 genes, we are interested to identify the colorectal cancer associated genes on the frequently amplified $q$ arm of chromosome $20 .{ }^{[3-5,11]}$ The increasing frequency of amplification from sporadic adenomas to carcinomas and the presence of this amplification in carcinomas of other tissue origin suggests that $20 \mathrm{q}$ region harbors one or more proto-oncogene(s) whose amplification is a defining event in the colorectal tumorigenesis and progression. The probability that an amplified gene could lead to tumorigenesis is supported by several examples [12-15]. There are over 400 genes located on the $q$ arm of chromosome 20 , thus the challenge is identifying which gene or genes on 20 q may contribute to tumor initiation and/or progression. Previously, most studies of chromosomal amplification have been done using fluorescence in situ hybridization (FISH) and/or comparative genomic hybridization $(\mathrm{CGH})$ technique performed on metaphase chromosomes. While the data from FISH and CGH to metaphase chromosomes identifies broad genomic regions in which there is amplification, the resolution is low (at best $1 \mathrm{Mb}$ ) and coverage of the amplicon non-continuous. The development of microarray technology and exploitation of the technology by comparative genomic hybridization to arrayed genomic clones affords a higher resolution and broader examination of genomic regions than previously available. ${ }^{[16]}$

Using the BAC/PAC genomic microarray to scan for amplification has afforded an increased sensitivity necessary to provide a high-resolution map of the $20 q$ amplicon detected in the colorectal cancer cell lines, we examined. The validation experiments with breast carcinoma cell lines MCF7 and SKBr3, when compared with previous studies, provided mixed results. ${ }^{[9,10]}$ For $\mathrm{SKBr} 3$, the genomic profile generated with both array 1 and array 2 were consistent and agreed with regions of amplification previously identified using $\mathrm{FISH}^{\left[{ }^{[9]}\right.}$ and BAC array-CGH. ${ }^{[10]}$ However, although MCF7's genomic profile was consistent from array 1 to array 2 and agreed with the previous FISH study, ${ }_{,}^{[9]}$ the profile was not consistent with that seen in the previous BAC array-CGH

Table 1: Candidate Amplified and Over Expressed Genes

\begin{tabular}{|c|c|c|}
\hline Gene Name & Position & Description \\
\hline C20orf24 & 35872566 & Protein of unknown function \\
\hline RPN2 & 36445899 & $\begin{array}{l}\text { Ribophorin II, a subunit of oligosaccharyltransferase, localizes to the rough endoplasmic reticulum and } \\
\text { binds ribosomes }\end{array}$ \\
\hline CTNNBL1 & 36960837 & Protein of unknown function, has moderate similarity to uncharacterized C. elegans M01E11.2 \\
\hline MYBL2 & 42934133 & $\begin{array}{l}\text { Myeloblastosis oncogene-like } 2 \text {, transcription factor associated with promotion of DNA synthesis and cell } \\
\text { cycle progression, involved in the regulation of cell survival, proliferation, and differentiation }\end{array}$ \\
\hline YWHAB & 44152760 & $\begin{array}{l}\text { 14-3-3 beta, mediate signal transduction by binding to phosphorylated serine residues on a variety of sig } \\
\text { naling molecules }\end{array}$ \\
\hline UBE2C & 45079659 & Ubiquitin-conjugating enzyme E2C, binds anaphase-promoting complex subunit 2 (APC2) \\
\hline DPM1 & 49734039 & $\begin{array}{l}\text { Dolichol phosphate mannose synthase 1, catalytic subunit that complexes with DPM2 and DPM3 to form } \\
\text { Dol-P-Man synthase }\end{array}$ \\
\hline ADNP & 50005914 & Activity-dependent neuroprotective protein, a putative transcription factor \\
\hline PFDN4 & 53463042 & $\begin{array}{l}\text { Prefoldin } 4 \text {, part of a heterohexameric chaperone complex involved in tubulin and actin folding, putative } \\
\text { transcription factor that may function during the G1 to S phase transition }\end{array}$ \\
\hline C20orf43 & 55682105 & Protein of unknown function \\
\hline
\end{tabular}


study. ${ }^{[10]}$ The difference in the profiles generated could be due to the documented high variation in MCF7 cell lines from different sources. ${ }^{[17]}$ Our source for MCF7 was ATCC, and based on their karyotype information as well as that previously published, MCF7 has been reported with a range of chromosome 20 copy number, from being nullisomic to polysomic. ${ }^{[17,18]}$ The profile generated with our genomic array is consistent with that reported in the SKY, FISH, CGH database on The University of Cambridge Cancer Genomics Program website. ${ }^{[19]}$

The genomic profiles for HT29 and CaCo-2 revealed an overall increase of one and two copies of chromosome $20 q$ respectively, which is in agreement with previous karyotyping and metaphase CGH studies. ${ }^{[20,21]}$ However, with the custom array-CGH we were able to detect further regional gains at the $37-39 \mathrm{Mb}, 42-45 \mathrm{Mb}$, $48-50 \mathrm{Mb}$, and $52-56 \mathrm{Mb}$ intervals. The HT29 profile also reveals a distinct copy gain at $53 \mathrm{Mb}$, while CaCo-2 appears to have a distinct copy gain at $43 \mathrm{Mb}$. Overall, HT29's profile shows fewer distinct copy gains while CaCo-2 appears to have multiple distinct copy gains. When these genomic profiles were combined with expression array data for over 200 genes on 20q, candidate genes in amplified regions were identified. The fact that, of the 23 genes for HT29 and 34 genes for CaCo2 whose probes showed significant $(p<0.01)$ increased expression, 21 were common to both cell lines suggests a common expression signature for the cell lines. When both the location of the 21 genes with respect to the common intervals of copy gain and the function of the gene products was considered, 10 genes were identified for further examination as candidate genes associated with colorectal cancer.

Of the 10 candidate genes, the open reading frames (C20orf24 and C20orf43) and CTNNBL1 are novel and uncharacterized genes whose possible role in cancer is, therefore, unknown. The gene RPN2 is located in the proximal region of the contig and its protein functions as a subunit of proteosomes regulatory complex 19S. This subunit has recently been reported to be the target of caspase cleavage upon induction of apoptosis, thus an over expression of the gene may function in increasing resistance to apoptosis in cancer cells. ${ }^{[22]}$ The candidate genes MYBL2 and UBE2C are directly involved in cell cycle progression through the promotion of $\mathrm{G} 1$ to $\mathrm{S}$ phase and the degradation of cyclins as part of the APC2 complex respectively, thus their importance in cancer cells has been previously noted. ${ }^{[23,24]}$ According to a previous study, the protein encoded by ADNP may increase the viability of certain cell types through modulation of p53 activity, thus making it a very attractive oncogene candidate as well. ${ }^{[25]}$ YWHAB coeds for the protein 14-3-3 beta, which is involved in mediating signal transduction as well as having been shown to promote cell spreading and migration through it's interaction with beta-1 integrin. ${ }^{[26]}$ Thus it is likely that over expression of YWHAB not only may perturb regulatory signal transduction pathways but also may promote tumor cell invasion and metastasis. While the general function of DPM1, as a subunit of the Dol-P Man synthase, is known to involve protein modification of $\mathrm{N}$-glycans and the proper surface expression of GPI-anchored proteins, it's possible role in cancer is unknown. One could suppose over expression of DPN1 may alter the number and type of GPI-anchored proteins on the cell surface, affecting cell cell interaction, adhesion and cellular transport. The gene prefoldin 4, PFDN4, resides on the distinct copy gain at 53Mb in HT-29, and also happens to be significantly over expressed in both cell lines. In one published study, the chaperonin protein coded for by PFDN4 showed cell cycle specific expression and was highly expressed in cells in crisis, suggesting that PFDN4 may play an important role in cell cycle regulation and overcoming senescence ${ }^{[27]}$. Interestingly, the highly amplified region at $43 \mathrm{Mb}$ in $\mathrm{CaCo}-2$ did not contain a candidate gene, but MYBL2 and YWHAB flank this region and could be affected by this amplification.

Utilizing both array-CGH and expression array analysis, a list of over 200 potential candidate genes represented on the arrays was efficiently reduced to 10 candidate genes for further examination. Other commonly amplified genomic regions in colon and breast tumors include 8p11-12, 17q23 and 12q13-14 [14,28,29]. In utilizing microarray technology, identification of amplicon genes contributing to tumor progression can be done in a genome wide, efficient manner. These genes could serve to identify the cellular pathways commonly altered in human cancer and signify which profiles are associated with certain tumor phenotypes. The genes identified could also be used as a panel of tumor markers for 
diagnosis, prognosis as well as identification of targets for drug therapy effective in the treatment of tumors.

\section{Acknowledgements}

The authors would like to acknowledge the collaborative efforts of Zhenyu Yuan PhD and Kun Zhang PhD in producing the custom microarrays. The authors would also like to acknowledge the contribution of technical expertise by Scott Vacha, PhD in standardizing hybridization procedures.

\section{References}

1. Hanahan D, Weinberg RA. Hallmarks of cancer, Cell 2000;100:57-70.

2. Albertson DG, Collins C, McCormick F, Gray JW. Chromosome aberrations in solid tumors, Nature Genetics 2003;34:369-76.

3. Hidaka S, Yasutake T, Takeshita H, Knodo M, Nanashima A, Tagawa $Y$, et al. Differences in 20q13.2 copy number between colorectal cancers with and without metastasis, Clinical Cancer Research 2000;6:2712-7.

4. Al-Mulla F, Keith WN, Pickford IR, Going JJ, Birnie GD. Comparative Genomic hybridization analysis of primary colorectal carcinomas and their synchronous metastasis, Genes, Chromosomes \& Cancer 1999;24:306-14.

5. Aragane H, Sakakura C, Nakanishi M, Yasuoka R, Fujita $\mathrm{Y}$, Yamagishi $\mathrm{H}$, et al. Chromosomal aberrations in colorectal cancers and liver metastases analyzed by comprarative genomic hybridization, International Journal of Cancer 2001;94:623-9.

6. Marra MA, Kucaba TA, Dietrich NL, Green ED, Brownstein $\mathrm{B}$, Waterston $\mathrm{RH}$, et al. High-throughput fingerprint analysis of large-insert clones, Genome Research 1997;7:1072-84.

7. http://www.ncbi.nlm.nih.gov/

8. Fiegler H, Carr P, Douglas EJ, Burford DC, Hunt S, Carter NP, et al. DNA microarrays for comparative genomic hybridization based on DOP-PCR amplification of BAC and PAC clones, Genes, Chromosomes \& Cancer 2003;36:361-74.

9. Tanner M, Tirkkonen M, Kallionieme A, Collins C, Hintz M, Gray JW, et al. Increased copy number at 20 q13 in breast cancer:defining the critical region and exclusion of candidate genes, Cancer Research 1994;54:4257-60.

10. Lapuk A, Volik S, Vincent R, Chin K, Kuo W, Gray JW, et al. Computational BAC clone contig assembly for comprehensive genome analysis, Genes, Chromosomes \& Cancer 2004;40:66-71.

11. Meijer GA, Hermsen MA, Baak JP, van Diest PJ, Meuwissen SG, Walboomers JM, et al. Progression from colorectal adenoma to carcinoma is associated with nonrandom chromosomal gains as detected by comparative genomic hybridization, Journal of Clinical Pathology, 1998;51:901-9.

12. Mahdy E, Pan $Y$, Wang N, Malmstrom PU, Ekman $P$, Bergerheim U. Chromosome 8 numerical aberration and
C-MYC copy number gain in bladder cancer are linked to stage and grade, Anticancer Research 2001;21:3167-73.

13. Redon R, Hussenet T, Bour G, Caulee K, Jost B, Muller $D$, et al. Amplicon mapping and transcriptional analysis pinpoint $C y c l i n ~ L$ as a candidate oncogene in head and neck cancer, Cancer Research 2002;62:6211-7.

14. Nathanson DR, Culliford AT 4th, Shia J, Chen B, D'Alessio M, Zeng ZS, Paty PB, et al., HER 2/neu expression and gene amplification in colon cancer, Int $\mathrm{J}$ Cancer 2003;105:769-802.

15. Kuchiki H, Yasuda J, Kayama T, Murakami Y, Sekiya T. Detection of DNA abnormalities by arbitrarily primed PCR fingerprinting:amplification of the MDM2 gene in a mediastinum fibrosarcoma, Biochem Biophys Res Commun 1999;258:271-7.

16. Solinas-Toldo S, Lampel S, Stilgenbauer S, Nickolenko $J$, Benner A, Dohner $\mathrm{H}$, et al. Matrix-based comparative Genomic Hybridization:biochips to screen for genomic imbalances, Genes, Chromosomes \& Cancer 1997;20:399407.

17. Osborne CK, Hobbs K, Trent JM. Biological differences among MCF-7 human breast cancer cell lines from different laboratories, Breast Cancer Res Treat 1987;9:11121.

18. http://www.atcc.org/

19. http://www.path.cam.ac.uk/ pawefish/index.html

20. Kawai K, Viars C, Arden K, Tarin D, Urquidi V, Goodison S. Comprehensive karyotyping of the HT-29 colon adenocarcinoma cell line, Genes, Chromosomes \& Cancer 2002;34:1-8

21. Bezrookove V, Smits R, Moeslein G, Fodde R, Tanke HJ, Raap AK, et al. Premature chromosome condensation revisited:a novel Chemical approach permits efficient cytogenetic analysis of cancers, Genes, Chromosomes \& Cancer 2003;38:177-86.

22. Sun XM, Butterworth M, MacFarlane M, Dubiel W, Ciechanover A, Cohen GM. Caspase activation inhibits proteasome function during apoptosis, Molecular Cell 2004;14:81-93.

23. Oh IH, Reddy EP. The myb gene family in cell growth, differentiation and apoptosis, Oncogene 1999;18:301733.

24. Tang Z, Li B, Bharadwaj R, Zhu H, Ozkan E, Hakala K, et al. APC2 Cullin protein and APC11 RING protein comprise the minimal ubiquitin ligase module of the anaphasepromoting complex, Mol Biol Cell 2001;12:3839-51.

25. Zamostiano R, Pinhasov A, Gelber E, Steingart RA, Seroussi E, Brenneman DE, et al. Cloning and characterization of the human activity-dependent neuroprotective protein, J Biol Chem 2001;276:708-14.

26. Rodriguez LG, Guan JL. 14-3-3 regulation of cell spreading and migration requires a functional amphipathic groove, J Cell Physiol. 2005;202:285-94.

27. lijima M, Kano Y, Nohno T, Namba M. Cloning of cDNA with possible transcription factor activity at the G1-S phase transition in human fibroblast cell lines, Acta Med Okayama 1996;50:73-7.

28. Yanga Z, Albertso D, Ethier SP. Genomic organization of the 8p11-p12 amplicon in three breast cancer cell lines, Cancer Genetics and Cytogenetics 2004;155:57-62.

29. Monni O, Barlund M, Mousses S, Kononen J, Sauter G, Heiskanen $M$, et al. Comprehensive copy number and gene expression profiling of the 17q23 amplicon in human breast cancer. Proc Natl Acad Sci 2001;98:5711-6. 